Synthesis Alerts is a monthly feature to help readers of Synthesis keep abreast of new reagents, catalysts, ligands, chiral auxiliaries, and protecting groups which have appeared in the recent literature. Emphasis is placed on new developments but established reagents, catalysts etc are also covered if they are used in novel and useful reactions. In each abstract, a specific example of a transformation is given in a concise format designed to aid visual retrieval of information.

Synthesis Alerts is a personal selection by:

Elyse Bourque, Jennifer Delaney, Andrew Gunn, Stephen McAteer, Marcel de Puit, Sukhjinder Uppal, Tanya Wildmann and Josephine Yuen, Department of Chemistry, Leeds University, Leeds, LS2 9JT, UK.

Georg Thieme Verlag does not accept responsibility for the accuracy, content, or selection of the data.
The journals regularly covered by the abstractors are:

Advanced Synthesis and Catalysis

Angewandte Chemie

Chemical Communications

Chemistry-A European Journal

Collection of Czechoslovak Chemical Communications

European Journal of Organic Chemistry

Helvetica Chimica Acta

Journal of Organic Chemistry

Journal of the American Chemical Society

Organic Letters

Organometallics

Perkin Transactions 1

Synlett

Synthesis

Tetrahedron

Tetrahedron Asymmetry

Tetrahedron Letters

Synthesis 2002, No. 7, 2105 2002. Article Identifier:

1437-210X,E;2002,0,07,973,980,ftx,en;X00702SS.pdf.

(c) Georg Thieme Verlag Stuttgart · New York

ISSN 0039-7881

Polymer supported catalytic enantioselective olefin metathesis. Hultzsch, K. C.; Jernelius, J. A.; Hoveyda, A. H.; Schrock, R. R. Angew. Chem. Int. Ed. 2002, 41, 589.

Enantioselective Olefin Metathesis
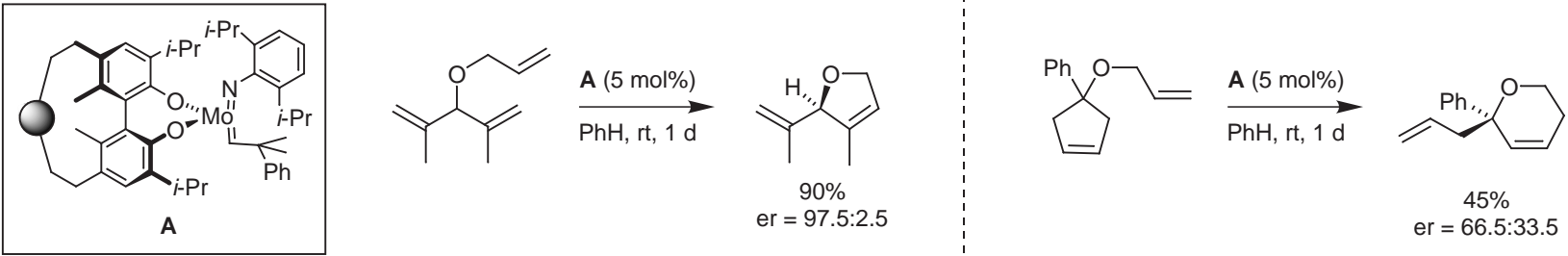

8 examples (yields $26-92 \%$, \%ee 33-98\%). Synthesis and recycling of the catalyst are also reported.

Fe-catalysed cross coupling.

Fürstner, A.; Leitner, A. Angew. Chem. Int. Ed. 2002, 41, 609.

$\mathrm{sp}^{2}-\mathrm{sp}^{3}$ Coupling
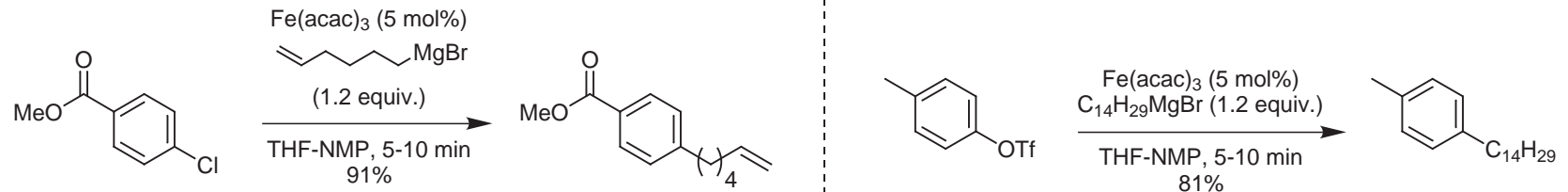

38 examples (yields 0-96\%).

Synthesis of protected syn 1,3-diols.

Grimaud, L.; Mesmay, R.; Prunet, J. Org. Lett. 2002, 4, 419.

Diastereoselective 1,4-Addition

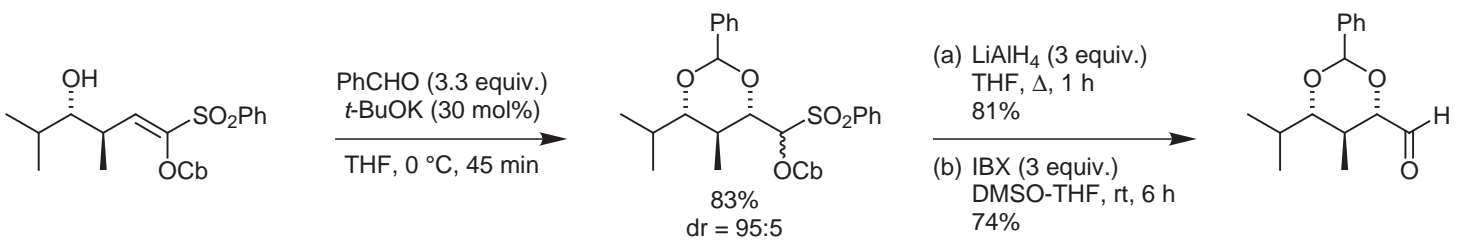


Ligand-accelerated, copper-catalyzed asymmetric hydrosilylations of aryl ketones.

Lipshutz, B. H.; Noson, K.; Chrisman, W. J. Am. Chem. Soc. 2001, 123, 12917.

Asymmetric 1,2-Addition
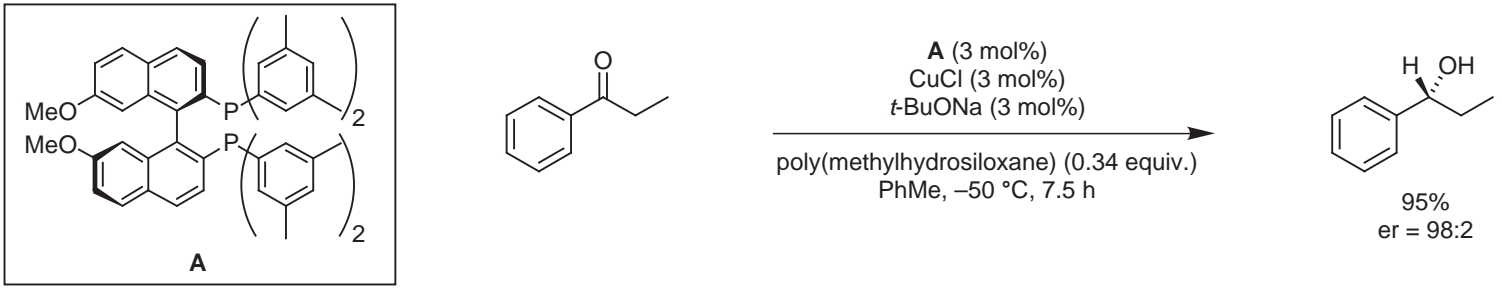

10 Examples (yields $87-99 \%$, \%ee 67-97\%)

Vanadium-catalyzed aldol reaction of allenic alcohols and aldehydes.

Trost, B. M.; Jonasson, C.; Wuchrer, M. J. Am. Chem. Soc. 2001, 123, 12736.

1,2-Addition

(1.2 equiv.

15 Examples (yields 57-88\%).

Synthesis of 1,7-dioxaspiro[5.5]undecanes

Keller, V. A.; Martinelli, J. R.; Strieter, E. R.; Burke, S. D. Org. Lett. 2002, 4, 467.

Ring Closing Metathesis/Spiroketalisation


7 examples of acid-catalysed spiroketalisation (yields $71-97 \%$ ).

Highly regioselective and diastereoselective directed hydroformylation of allylic ethers.

Krauss, I. J.; Wang, C. C.-Y.; Leighton, J. L. J. Am. Chem. Soc. 2001, 123, 11514.

Hydroformylation<smiles></smiles>



\begin{tabular}{llll}
$\mathrm{R}^{1}$ & $\mathrm{R}^{2}$ & Yield & $\%$ de \\
\hline $\mathrm{Me}$ & $\mathrm{H}$ & $92 \%$ & $91 \%$ \\
$\mathrm{Ph}$ & $\mathrm{H}$ & $96 \%$ & $93 \%$ \\
$i-\mathrm{Pr}$ & $\mathrm{H}$ & $94 \%$ & $97 \%$ \\
$\mathrm{H}$ & $\mathrm{Me}$ & $88 \%$ & ----
\end{tabular}<smiles></smiles>

6 Examples (yields $87-98 \%$, \%de $91-97 \%$ ).

$94 \%$

$\mathrm{dr}=85: 15$

Asymmetric Amination/Alkylation

Synthesis of 1,2-amino alcohols via a direct asymmetric three-component Mannich reaction. List, B.; Pojarliev, P.; Biller, W. T.; Martin, H. J. J. Am. Chem. Soc. 2002, 124, 827.



$83 \%$

$\mathrm{dr}=9: 1$

er $=97: 3$

8 examples (yield 57-92\%, \%ee 61-99\%, dr 3:1 $\rightarrow 20: 1$ ). Various ketones and aldehydes were used 
Enantioselective Staudinger synthesis of $\beta$-lactams catalyzed by a planar-chiral nucleophile. Hodous, B. L.; Fu, G. C. J. Am. Chem. Soc. 2002, 124, 1578.

[2+2] Enantioselective Cycloaddition

<smiles>O=C1CCCCC1</smiles>


$84 \%$ er $=91: 9$<smiles>CCC(=C=O)c1ccccc1</smiles><smiles>[Sn]=CC1CC1</smiles><smiles>CC(C)COC(=O)OCC(C)C</smiles>

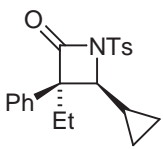

1.15 equiv.

13 examples (yield $82-98 \%$, \%ee $81-98 \%$ )

Indium(III) chloride-sodium borohydride system as an alternative to tributyltin hydride. Inoue, K.; Sawada, A.; Shibata, I.; Baba, A. J. Am. Chem. Soc. 2002, 124, 906.

Radical Reduction/Addition
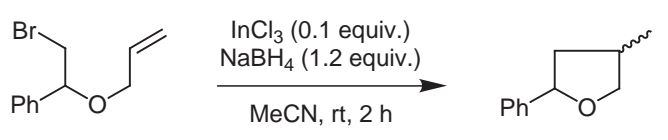

$50 \%$

cis:trans $=15: 85$


5 equiv.

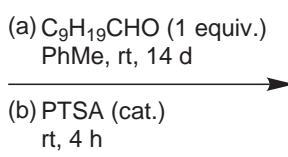

1.5 equiv.

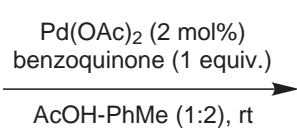

$55 \%$

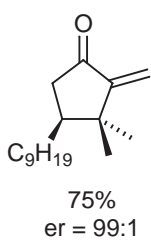

Selective Pd-catalyzed oxidative coupling of anilides with olefins through $\mathrm{C}-\mathrm{H}$ bond activation.

Boele, M. D. K.; van Strijdonck, G. P. F.; de Vries, A. H. M.; Kamer, P. C. J.; de Vries, J. G.; van Leeuwen, P. W.

$s p^{2}-s p^{2}$ Coupling

N. M. J. Am. Chem. Soc. 2002, 124, 1586.<smiles>O=C(Nc1ccccc1)c1ccccc1</smiles>

$>\mathrm{CO}_{2} \mathrm{Bu}$

1.1 equiv. vinyl acetate (3 equiv.)<smiles>OC12CC3CC(CC(C3)C1)C2</smiles>
$\underset{\mathrm{Na}_{2} \mathrm{CO}_{3}(60 \mathrm{~mol} \%)}{\mathrm{PhMe}, 100{ }^{\circ} \mathrm{C}, 2 \mathrm{~h}}$
$\stackrel{\text { IrCl(Cod }]_{2}(1 \mathrm{~mol} \%)}{\longrightarrow}$ $75 \%$

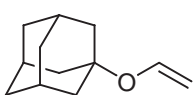<smiles>Oc1ccc(O)cc1</smiles>

vinyl acetate (4 equiv.)

$[\mathrm{IrCl}(\mathrm{cod})]_{2}(1 \mathrm{~mol} \%)$

$\mathrm{NaOAc}$ (1.2 equiv.)

PhMe, $100{ }^{\circ} \mathrm{C}, 2 \mathrm{~h}$ $90 \%$

$$
\mathrm{HO}
$$

Stereocontrolled synthesis of $\alpha$-exomethylene $\gamma$-lactones with a stereogenic quaternary carbon centre.

$(-)-8-\left(\right.$ phenyl)menthoxy $=R^{\prime}$ 
Asymmetric synthesis of cetirizine dihydrochloride.

Pflum, D. A.; Krishnamurthy, D.; Han, Z.; Wald, S. A.; Senanayake, C. H. Tetrahedron Lett. 2002, 43, 923.

Asymmetric Addition

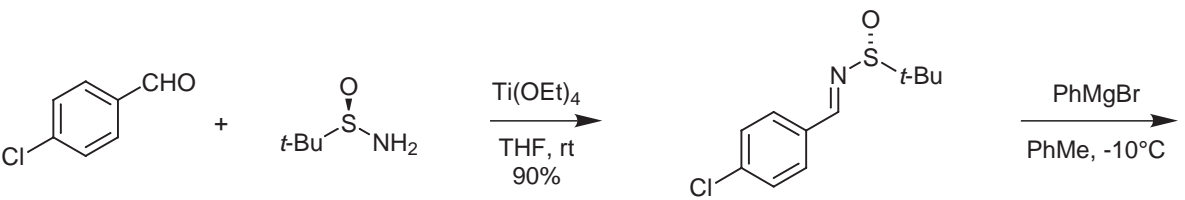<smiles>CC(C)(C)S(=O)NC(c1ccccc1)c1ccc(Cl)cc1</smiles>

The opposite enantiomer can be synthesised as the major product by starting with benzaldehyde and adding 4-chlorophenylmagnesium bromide. The remaining steps in the synthesis of cetirizine dihydrochloride are also reported.

Stereocontrolled synthesis of (-)-macrolactin A.

Marino, J. P.; McClure, M. S.; Holub, D. P.; Comasseto, J. V.; Tucci, F. C. J. Am. Chem. Soc. 2002, 124, 1664.
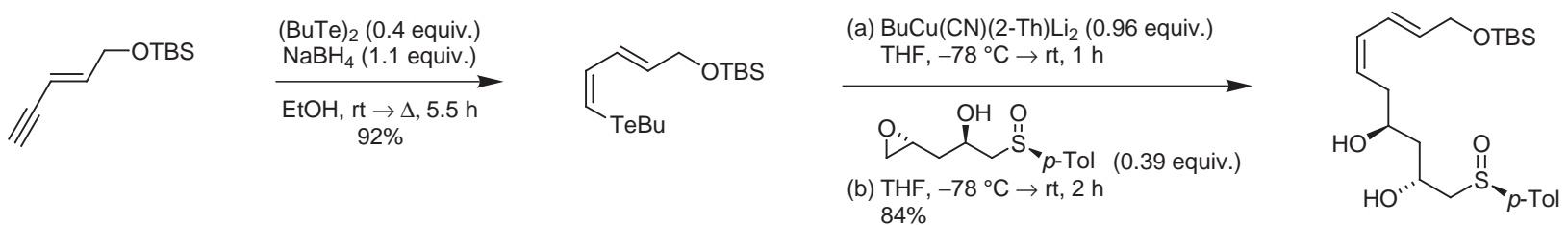

This is the first reported use of vinyl tellurides in the synthesis of a natural product.

Nickel-catalyzed cross-coupling of alkylzinc halides and alkyl halides.

Jensen, A. E.; Knochel, P. J. Org. Chem. 2002, 67, 79.<smiles>O=C(CCCI)c1ccccc1</smiles>
$+$



$69 \%$ Hydrometalation/Alkylation 
<smiles>CC(C)c1ccccc1I</smiles><smiles>CCOC(=O)CC(=O)OCC</smiles><smiles>OC1C=CC(=Cc2ccccc2)C=CC1</smiles>
Cul (10 mol\%)

$\mathrm{Cs}_{2} \mathrm{CO}_{3}(1.5$ equiv.) THF, $\Delta, 31 \mathrm{~h}$

16 examples (yields 61-98\%)
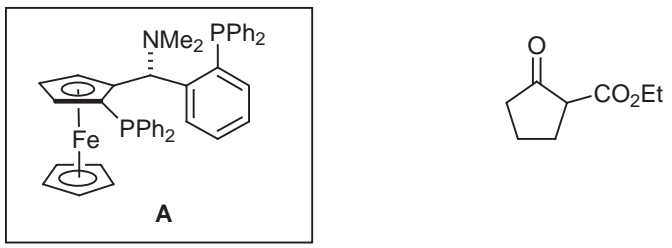

$$
\begin{gathered}
\mathbf{A}(0.9 \mathrm{~mol} \%) \\
\underset{\mathrm{EtOH} / \mathrm{CH}_{2} \mathrm{Cl}_{2}(1: 10), 50{ }^{\circ} \mathrm{C}, 63 \mathrm{~h}}{\stackrel{\left.\mathrm{Ru}(\mathrm{cod})\left(\mathrm{C}_{4} \mathrm{H}_{7}\right)_{2}\right] \mathrm{HBr}(0.8 \mathrm{~mol} \%)}{\longrightarrow}}
\end{gathered}
$$<smiles>CCOC(=O)C(C(=O)OCC)c1ccccc1C(C)(C)C</smiles>

Examples of hydrogenation of $\alpha$-(acylamino)acrylic acids, enol esters, enamides, 1,3-diketones and hydrazones using Rh- or Ru-catalysts with ferrocenylphosphanes.

Cyclopentannulation of enones with acetal or orthoester organocuprates Ding, P.; Ghosez, L. Tetrahedron, 2002, 58, 1565.

1,4-Addition/Cyclization

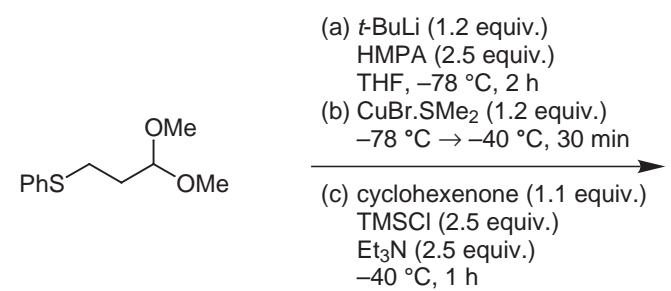<smiles>COC1=CC([C@@H]([SnH3])CC(OC)OC)CCC1</smiles>

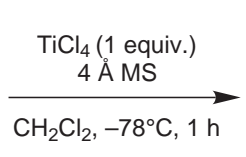

$\mathrm{Et}_{3} \mathrm{~N}(2.5$ equiv.
$-40^{\circ} \mathrm{C}, 1 \mathrm{~h}$

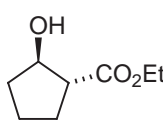

er $=95: 5$

$\mathrm{dr}=99: 1$

5 examples of acetal organocuprates (yields 40-93\%) and 1 example of an orthoester organocuprate.

Bicyclo[3.2.1] octan-8-ones by Pd-catalysed isomerisation of 2-vinyl-hexahydro-2,3-benzofurans.

Langer, P.; Holtz, E.; Saleh, N. N. R. Chem.-Eur. J. 2002, 8, 917.

Isomerization<smiles>O=C1CCCCC1S(=O)(=O)O</smiles><smiles>O=C(O)CC=CCBr</smiles><smiles>C=C[C@H]1CC2=C(S(=O)(=O)Oc3ccccc3)CCC[C@H]2O1</smiles>

$45 \%$

$\mathrm{dr}>98: 2$

\section{[Pd(dppe) $\left.)_{2}\right](5 \mathrm{~mol} \%)$} DMSO, $60^{\circ} \mathrm{C}, 24 \mathrm{~h}$

$\mathrm{DMSO}, 60^{\circ} \mathrm{C}, 24 \mathrm{~h}$



$81 \%$

endo:exo $>98: 2$

10 examples of bicyclo[3.2.1]octan-8-ones. High endo:exo selectivity obtained for the Pd-catalysed rearrangement with $\beta$-ketosulfones, but poor stereoselectivity for $\beta$-ketoesters.

Enhancement of methyltrioxorhenium-catalyzed epoxidation of alkenes by hexafluoro-2-propanol.

Iskra, J.; Bonnet-Deplon, D.; Bégué, J. P. Tetrahedron Lett. 2002, 43, 1001.

Epoxidation
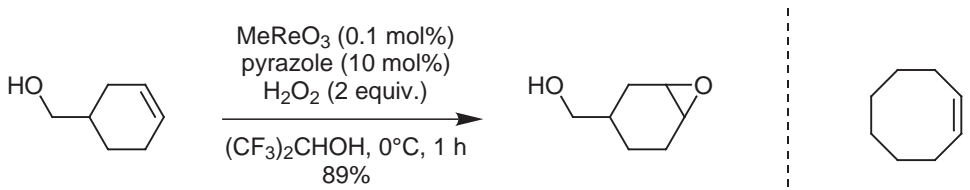

$\mathrm{MeReO}_{3}(0.1 \mathrm{~mol} \%)$

pyrazole (10 mol\%)

$\underset{\left(\mathrm{CF}_{3}\right)_{2} \mathrm{CHOH}, 0^{\circ} \mathrm{C}, 1 \mathrm{~h}}{\stackrel{\mathrm{H}_{2} \mathrm{O}_{2} \text { (2 equiv.) }}{\longrightarrow}}$

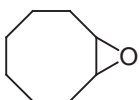

$93 \%$

7 examples (yields $80-93 \%$ ). 
Lithium N,O-dimethylhydroxyamide (LDHA) as an in situ protecting group for aromatic aldehydes.

Roschangar, F.; Brown, J. C.; Cooley Jr., B. E.; Sharp, M. J.; Matsuoka, R. T. Tetrahedron 2002, 58, 1657.

Protecting Group

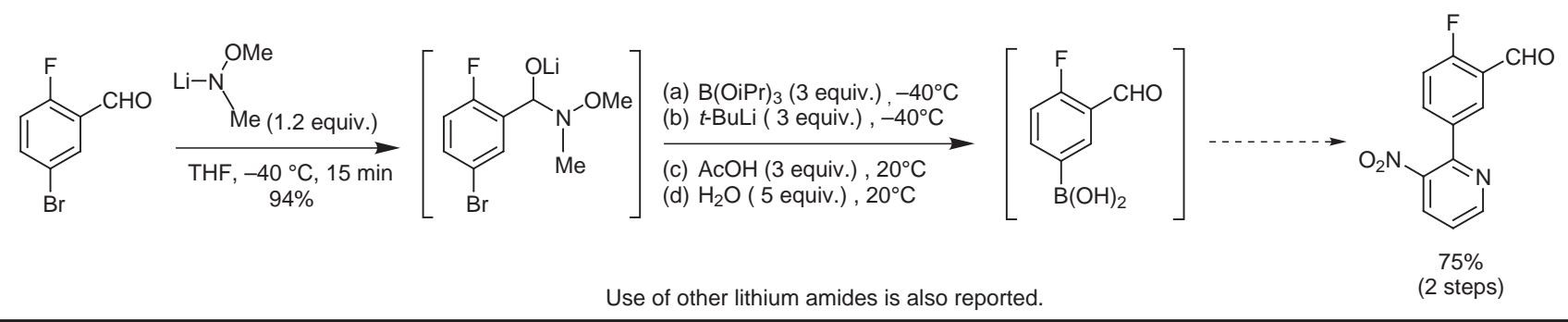

Stereoselective titanium-catalyzed hydroalumination of internal alkynes.

Stereoselective Reduction

Parenty, A.; Campagne, J. M. Tetrahedron Lett. 2002, 43, 1231.



6 examples (Yields 52-82\%, 9:1 Z/E $20: 1$ ).

Cross-coupling reaction of dianion cuprates with acid chlorides.

Ryu, I.; Ibeke, M.; Sonoda, N.; Yamato, S-Y.; Yamamura, G-H.; Komatsu, M. Tetrahedron Lett. 2002, 43, 1257.

Acylation


$41 \%$

(4 steps)

11 examples of 1,4 unsymmetrical diketones (Yields 40-76\%), including 2 examples of three-component coupling (Yields 41-59\%).

Copper-catalyzed indole formation from 2-ethynylaniline derivatives.

Hiroya, K.; Itoh, S.; Ozawa, M.; Kanamori, Y.; Sakamoto, T. Tetrahedron Lett. 2002, 43, 1277.

Heteroannulation

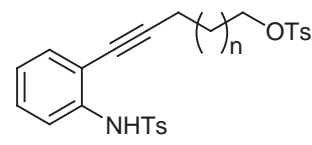
(a) $\mathrm{KH}$
$\left(\mathrm{CHCl}_{2}\right)_{2}, 0^{\circ} \mathrm{C} \varnothing \mathrm{rt}, 2 \mathrm{~h}$
(b) $\mathrm{Cu}(\mathrm{OAc})_{2}(50 \mathrm{~mol} \%)$
$\left(\mathrm{CHCl}_{2}\right)_{2}, \Delta, 48 \mathrm{~h}$

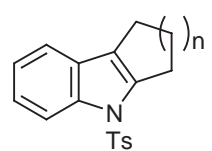

\begin{tabular}{cc}
$\mathrm{n}$ & Yield \\
\hline 1 & $67 \%$ \\
2 & $64 \%$
\end{tabular}

16 examples of indole cyclisation (Yields 9-95\%)

Preparation and utilization of nonracemic secondary $\alpha$-(carbamoyloxy)alkylzinc and copper reagents.

Papillon, J. P.; Taylor, R. J. K. Org Lett. 2002, 4, 119.
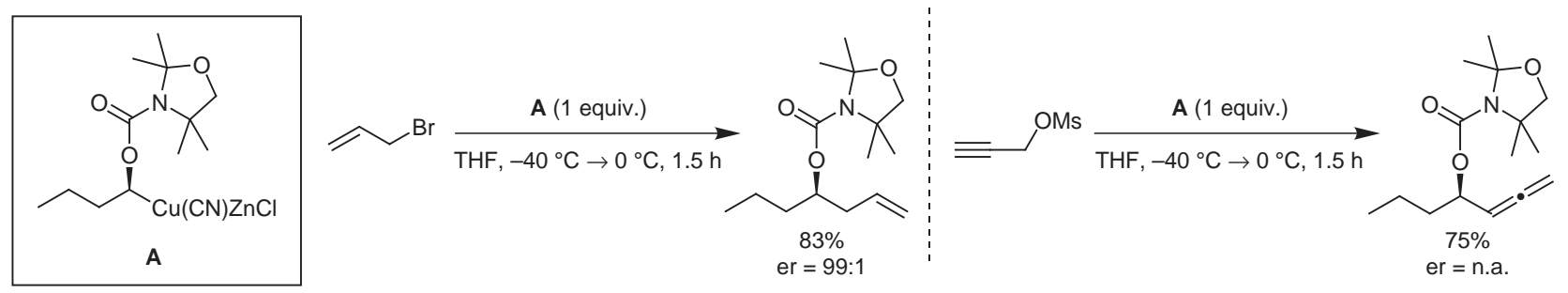

11 examples (yields $0-83 \%$ ) are reported as well as the synthesis of $\mathbf{A}$. 
Pinacol boronate synthesis catalyzed by a palladium/imidazolium salt system. Fürstner, A.; Seidel, G. Org Lett. 2002, 4, 541.

Borylation
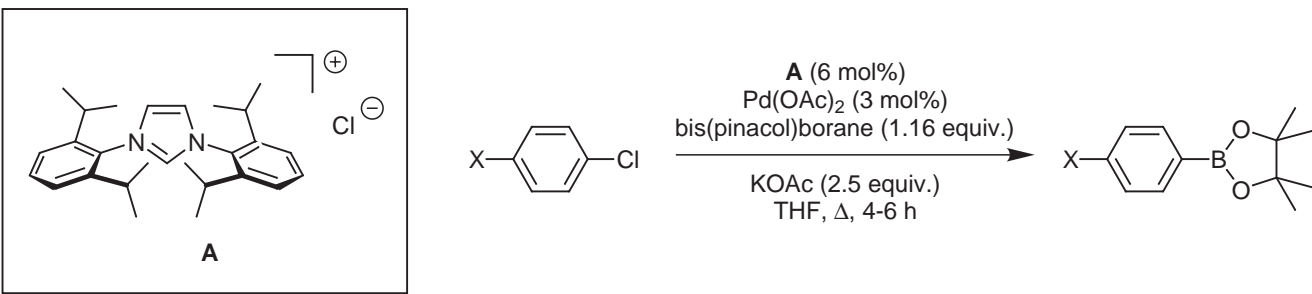

\begin{tabular}{ll}
$\mathrm{X}$ & Yield \\
\hline $\mathrm{CO}_{2} \mathrm{Me}$ & $85 \%$ \\
$\mathrm{CN}$ & $90 \%$ \\
$\mathrm{NO}_{2}$ & $63 \%$ \\
$\mathrm{CF}_{3}$ & $77 \%$
\end{tabular}

8 examples (yields 53-90\%).

Chelation-controlled 1,4-diastereoselective acylation/intramolecular acylation sequence.

Hirose, T.; Sunazuka, T.; Shirahata, T.; Yamamoto, D.; Harigaya, Y.; Kuawjima, I.; Omura, S. Org Lett.

2002, 4, 501.

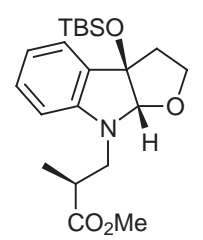
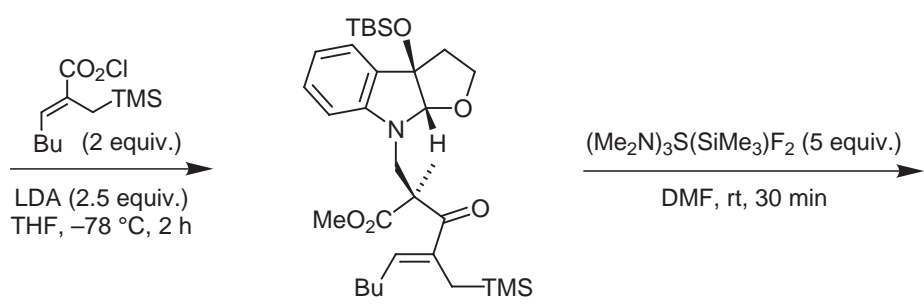

2 examples (yields $46-55 \%)$.

Diastereoselective Acylation

Substituted alkenes via migration-elimination reaction.

Kakiya, H.; Shinokubo, H.; Oshima, K. Tetrahedron 2001, 57, 10063.

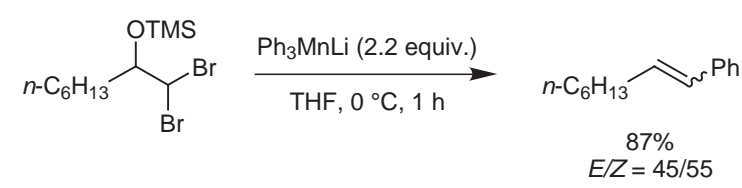

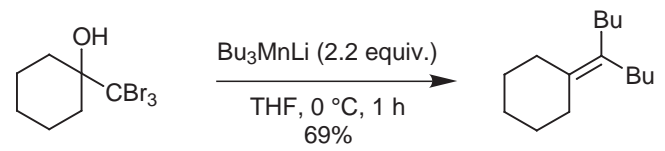

25 examples (yields 13-87\%, 69/31 E/Z 45/55).

Alkyl chlorides from alcohols using 2,4,6-trichloro[1,3,5]triazine and DMF.

De Luca, L.; Giacomelli, G.; Porcheddu, A. Org Lett. 2002, 4, 553.

Chlorination
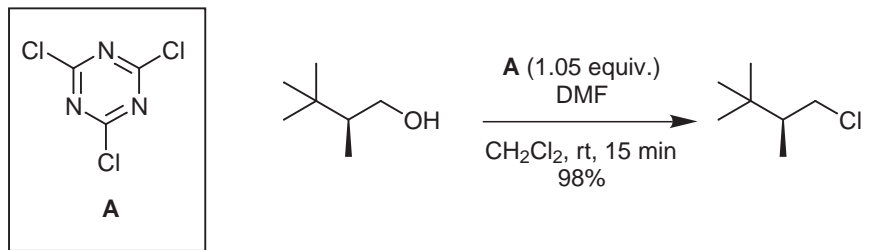<smiles>OC1CCCCC1O</smiles>

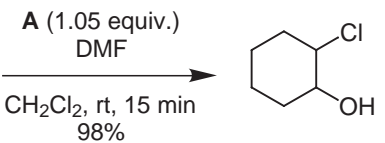

12 examples of aliphatic alcohols (yields 96-99\%), 3 examples of diols (yields 95-98\%), 2 examples of unsaturated alcohols (yields 92-98\%) and 6 examples of $\beta$-amino alcohols (yields $83-97 \%$ ).<smiles>O=C1CCC(/C=C/Br)C1</smiles>

$\mathrm{PhSi}(\mathrm{OMe})_{3}$ (2 equiv.) $\left[\mathrm{Rh}(\mathrm{cod})(\mathrm{MeCN})_{2}\right] \mathrm{BF}_{4}(2 \mathrm{~mol} \%)$ Dioxane $/ \mathrm{H}_{2} \mathrm{O}\left(\underset{82 \%}{10: 1)}, 90^{\circ} \mathrm{C}, 20 \mathrm{~h}\right.$

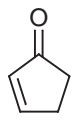

$\mathrm{PhSi}(\mathrm{OMe})_{3}$ (2 equiv.) $\underset{\text { Dioxane } / \mathrm{H}_{2} \mathrm{O}(10: 1), 90^{\circ} \mathrm{C}, 20 \mathrm{~h}}{\stackrel{\left[\mathrm{Rh}(\mathrm{cod})(\mathrm{MeCN})_{2}\right] \mathrm{BF}_{4}(2 \mathrm{~mol} \%)}{\longrightarrow}}$<smiles>O=C1CCC(c2ccccc2)C1</smiles> 
Catalytic asymmetric synthesis of $O$-acetyl cyanohydrins from potassium cyanide, acetic anhydride and aldehydes. Asymmetric Addition Belokon, Y. N. et al Chem. Commun. 2002, 244.
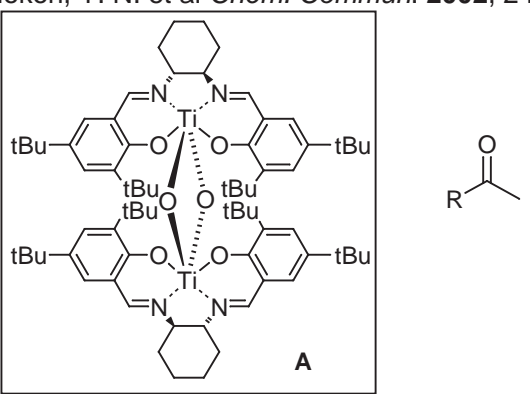
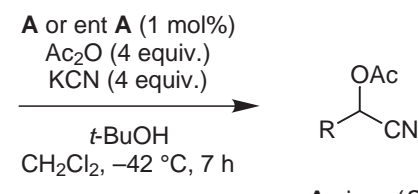

A gives $(S)$ ent $\mathbf{A}$ gives $(R)$

\begin{tabular}{lllll} 
R & $\begin{array}{l}\text { yield } \\
(\mathbf{A})\end{array}$ & $\begin{array}{l}\text { \%ee } \\
(\mathbf{A})\end{array}$ & $\begin{array}{l}\text { yield } \\
\text { (ent A) }\end{array}$ & $\begin{array}{l}\text { \%ee } \\
\text { (ent A) }\end{array}$ \\
\hline $\mathrm{Ph}$ & $93 \%$ & $90 \%$ & $92 \%$ & $89 \%$ \\
$p-\mathrm{FC}_{6} \mathrm{H}_{4}$ & $98 \%$ & $92 \%$ & $99 \%$ & $93 \%$ \\
$\mathrm{PhCH}_{2} \mathrm{CH}_{2}$ & $80 \%$ & $84 \%$ & $79 \%$ & $82 \%$ \\
$\mathrm{Me}_{2} \mathrm{CH}$ & $64 \%$ & $69 \%$ & $62 \%$ & $72 \%$
\end{tabular}

19 examples (yields $40-99 \%$, ee $60-93 \%$ ).

Chemo-, diastereo-, and enantioselective reduction of 1,2-dialkyl-3-aryl-1,3-diketones.

Ohtsuka, Y.; Koyasu, K.; Miyazaki, D.; Ikeno, T.; Yamada, T. Org. Lett. 2001, 3, 3421.

Enantioselective Reduction


\begin{tabular}{lllll}
$\mathrm{R}^{1}$ & $\mathrm{R}^{2}$ & yield & anti & $\%$ ee \\
\hline $\mathrm{Me}$ & $i-\mathrm{Pr}$ & $46 \%$ & $99 \%$ & $96 \%$ \\
$\mathrm{Et}$ & $i-\mathrm{Pr}$ & $41 \%$ & $99 \%$ & $98 \%$ \\
allyl & $i-\mathrm{Pr}$ & $47 \%$ & $98 \%$ & $96 \%$ \\
$\mathrm{Me}$ & $t-\mathrm{Bu}$ & $48 \%$ & $99 \%$ & $97 \%$ \\
$\mathrm{Me}$ & $\mathrm{C}_{9} \mathrm{H}_{19}$ & $47 \%$ & $98 \%$ & $96 \%$ \\
$\mathrm{Me}$ & sec-Bu & $47 \%$ & $98 \%$ & $95 \%$ \\
$\mathrm{Me}$ & $\mathrm{PhCH}_{2}$ & $45 \%$ & $94 \%$ & $98 \%$
\end{tabular}

Sodium borohydride is modified using ethanol and tetrahydrofurfuryl alcohol.

Chirality transfer in $\mathrm{Pd}(0)$-catalyzed coupling-cyclization of aryl iodides

Ma, S.; Shi, Z. Chem. Commun. 2002, 540.

Enantioselective Coupling-Cyclization

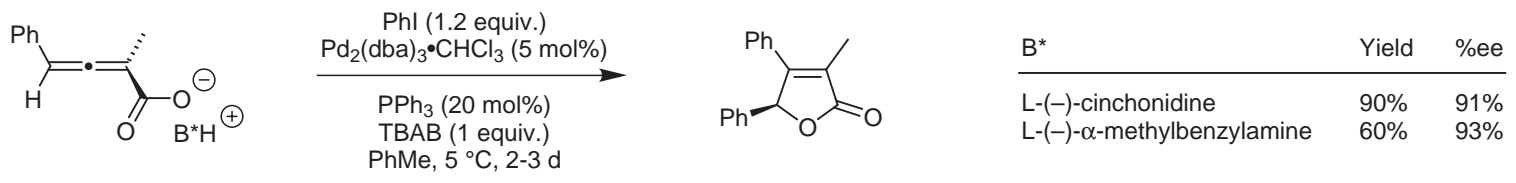

11 examples (yields 55-90\%, ee 91-98\%).

Asymmetric Michael addition of formaldehyde $N, N$-dialkylhydrazones to alkylidene malonates.

Vázquez, J.; Prieto, A.; Fernández, R.; Enders, D.; Lassaletta, J. M. Chem. Commun. 2002, 498.

Asymmetric Michael Addition
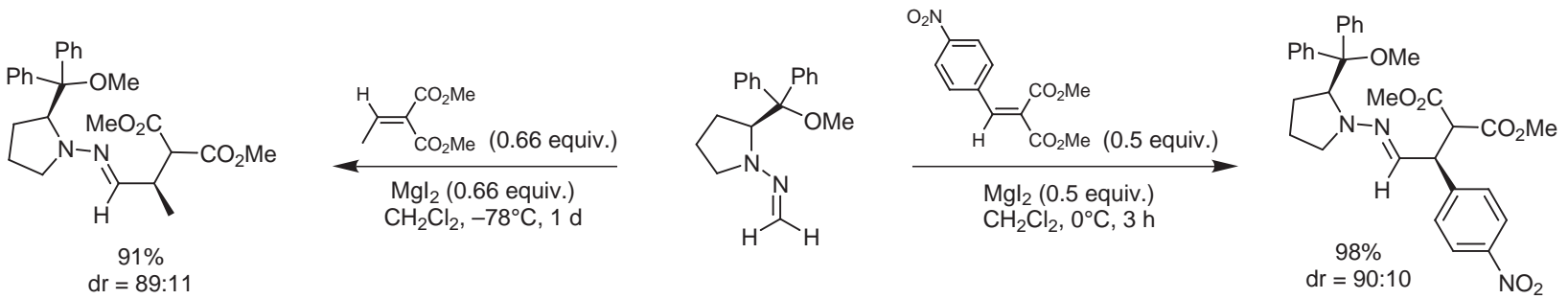

7 examples (yields $70-98 \%$, \%de 68-90\%).

1,2-Metallate rearrangement in the synthesis of D-erythro-sphingosine and D-erythro-ceramide.

Milne, J. E.; Jarowicki, K.; Kocienski, P. J.; Alonso, J. Chem. Commun. 2002, 426.

1,2-Metallate Rearrangement<smiles>O=S(=O)(O)C1C=C(O)C(O)CO1</smiles>



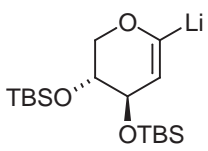

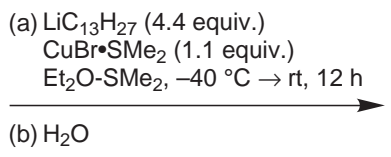

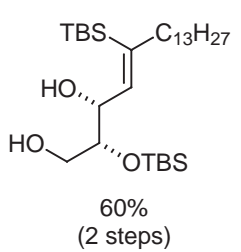

The remaining steps in the total synthesis of D-erythro-sphingosine are also reported. 